\title{
Molecular Gas and Star-formation in Selected H-ATLAS SDP Lensed SMGs
}

\author{
Lerothodi L. Leeuw ${ }^{1,2}$, Dominik A. Riechers ${ }^{3,4}$, John M. Carpenter ${ }^{3}$, \\ Mattia Negrello ${ }^{5}$, Rob J. Ivison ${ }^{6,7}$ and H-ATLAS Lensing and Core \\ ${ }^{1}$ College of Graduate Studies, UNISA, P. O. Box 392, UNISA, 0003, South Africa \\ ${ }^{2}$ SETI Institute, 189 Bernardo Avenue, Suite 100, Mountain View, CA 94043, USA \\ email: lerothodi@alum.mit.edu \\ ${ }^{3}$ Astronomy Department, California Institute of Technology, MC 249-17, 1200 East California \\ Boulevard, Pasadena, CA 91125, USA; ${ }^{4}$ Hubble Fellow \\ email: dr@astro.caltech.edu \\ ${ }^{5}$ Open University, Milton Keynes, MK7 6AA, United Kingdom \\ ${ }^{6}$ UK Astronomy Technology Centre, Royal Observatory, Blackford Hill, Edinburgh EH9 3HJ \\ ${ }^{7}$ Institute for Astronomy, University of Edinburgh, Blackford Hill, Edinburgh EH9 3HJ, UK
}

\begin{abstract}
We present detections of spatially resolved $\mathrm{CO}(J=2 \rightarrow 1)$ and $\mathrm{CO}(J=3 \rightarrow 2)$ emission, respectively, from the lensed submillimeter (submm) galaxies (SMGs), ID $9(z=1.577)$ and ID 17b $(z=2.308)$, found in the Science Demonstration Phase (SDP) of the Herschel Astrophysical Terahertz Large Area Survey (H-ATLAS, www.h-atlas.org). The detections were obtained using the Combined Array for Research in Millimeter-wave Astronomy (CARMA, www.mmarray.org) and confirm redshifts of the lensed galaxies. We exploit the CARMA data together with existing high- $J$ observations, to determine, among other physical properties of the lensed SMGs, the CO line luminosities, brightness temperature ratios, gas masses, and spatial sizes.
\end{abstract}

Keywords. galaxies: distances and redshifts — galaxies: ISM — gravitational lensing - molecular data - submillimeter

\section{Synopsis}

We summarize high significance $(>10 \sigma)$ detections of $\mathrm{CO}(J=2 \rightarrow 1)$ and $\mathrm{CO}(J=3 \rightarrow 2)$ line emission toward the lensed submillimeter (submm) galaxies (SMGs) ID $9(z=1.577)$ and ID 17b $(z=2.308)$, that were discovered in the Herschel Astrophysical Terahertz Large Area Survey (H-ATLAS, www.h-atlas.org). The detections were obtained with the Combined Array for Research in Millimeter-wave Astronomy (CARMA, www.mmarray.org) and are spatially resolved. From Gaussian fitting to the spatially integrated line profiles, we obtain $\mathrm{CO}(J=2 \rightarrow 1)$ and $\mathrm{CO}(J=3 \rightarrow 2)$ line peaks of $19.4 \pm 2.2 \mathrm{mJy}$ (ID 9$)$, $38.6 \pm 3.8 \mathrm{mJy}$ (ID 17b, component 1) and 13.1 $\pm 3.1 \mathrm{mJy}$ (ID 17b, component 2) with line FHWM of $371 \pm 52,157 \pm 26$ and $248 \pm 73 \mathrm{~km} \mathrm{~s}^{-1}$. From the fits, we also find redshifts of $1.5746 \pm 0.0002$ (ID 9), $2.3047 \pm 0.0001$ (ID 17b, component 1), and $2.3070 \pm 0.0003$ (ID 17b, component 2). The line parameters correspond to velocity-integrated emission line strengths of $7.63 \pm 0.91$ and $9.87 \pm 1.22 \mathrm{Jy} \mathrm{km} \mathrm{s}^{-1}$, and line luminosities of $L_{\mathrm{CO}(2-1)}^{\prime}=(2.48 \pm 0.29) \times 10^{11}\left(\mu_{\mathrm{L}}\right)^{-1} \mathrm{~K} \mathrm{~km} \mathrm{~s}^{-1} \mathrm{pc}^{2}$ and $L_{\mathrm{CO}(3-2)}^{\prime}=(2.85 \pm 0.35) \times$ $10^{11}\left(\mu_{\mathrm{L}}\right)^{-1} \mathrm{~K} \mathrm{~km} \mathrm{~s}^{-1} \mathrm{pc}^{2}$ (where $\mu_{\mathrm{L}}$ is the lensing magnification factor) for ID 9 and ID 17b, respectively. The detections will be used to estimate, among other physical properties, the total molecular gas masses, spatial sizes, and star-formation efficiencies of the SMGs and compared to those of similar objects in the context of evolutionary galaxy models. 